University of New Hampshire

University of New Hampshire Scholars' Repository

$11-2004$

\title{
Variable Bandwidth Filter for Multibeam Echo-sounding Bottom Detection
}

\author{
Barbara J. Kraft \\ University of New Hampshire, Durham \\ Christian de Moustier \\ University of California - San Diego
}

Follow this and additional works at: https://scholars.unh.edu/ccom

Part of the Computer Sciences Commons, and the Oceanography and Atmospheric Sciences and Meteorology Commons

\section{Recommended Citation}

B. J. Kraft and C. P. de Moustier, "Variable bandwidth filter for multibeam echo-sounding bottom detection," Oceans '04 MTS/IEEE Techno-Ocean '04 (IEEE Cat. No.04CH37600).

This Conference Proceeding is brought to you for free and open access by the Center for Coastal and Ocean Mapping at University of New Hampshire Scholars' Repository. It has been accepted for inclusion in Center for Coastal and Ocean Mapping by an authorized administrator of University of New Hampshire Scholars' Repository. For more information, please contact Scholarly.Communication@unh.edu. 


\title{
Variable bandwidth filter for multibeam echo-sounding bottom detection
}

\author{
B. J. Kraft and C. P. de Moustier \\ Center for Coastal and Ocean Mapping \\ NOAA Joint Hydrographic Center \\ University of New Hampshire \\ 24 Colovos Road \\ Durham, $\mathrm{NH} 03824$ USA \\ bjkraft@ccom.unh.edu, cpm@ccom.unh.edu
}

\begin{abstract}
The accuracy of a seafloor map derived from multibeam swath bathymetry depends first and foremost on the quality of the bottom detection process that yields estimates of the arrival time and angle of bottom echoes received in each beam. Filtering of each beam with a fixed bandwidth filter, with the bandwidth based on the length of the transmitted pulse, reduces the error associated with the time-angle estimates. However, filters of this type can not be optimal over the wide range of operational environments encountered. Better results are obtained with a processing scheme that varies the filter bandwidth across the swath width using detected time and angle information from the previous ping. This method is evaluated using sonar data obtained with a Reson SeaBat 8111ER and the results compared with those obtained using a fixed bandwidth filter.
\end{abstract}

\section{INTRODUCTION}

Bottom detection techniques applied to multibeam echo-sounding fall into three main categories: (1) a weighted mean time of arrival is estimated for each beam, (2) angles of arrival are estimated for each time increment through a beam deviation indicator algorithm, or (3) echo arrival time-angle pairs are derived from the zero crossing of phase in a split aperture correlator [1]. Various combinations of these techniques are implemented in most modern multibeam swath bathymetry sonars [2], [3], with refinements described by Yang and Taxt [4] and Gallaudet [5], as well as estimates of the associated errors (e.g., LeClerc [6] and Lurton [7]).

We focus here on a combination of methods (1) and (3), and we extend Gallaudet's [5] processing scheme which involves applying a maximum likelihood filter [8] to the noisy in-phase and quadrature components of the phasors obtained at the output of a split aperture correlator. The bandwidth of this maximum likelihood filter applied to each phasor was determined by setting the number of filter coefficients equal to the number of samples in 5 pulse lengths.

However, for any given beam, the duration of the received bottom echo depends on the beam width and the extent of its footprint on the bottom, itself a function of the range from the sonar to the bottom, the beam angle relative to vertical, and its incidence angle relative to the bottom. In addition, the signal to noise ratio of bottom echoes varies widely ( $>40 \mathrm{~dB}$ ) across the swath, due to the dependence of bottom acoustic backscatter on bottom type and angle of incidence. Therefore, no fixed bandwidth filter can be

This work performed under NOAA Grant No. NA170G2285 optimal over such a wide range of operating conditions.

In the approach presented here, the filter bandwidth, or cutoff frequency, is adjusted for each beam on a ping by ping basis. The filter coefficients of each beam are determined from information learned from the previous ping.

This method has been tested on data recorded with a Reson Seabat $8111 E R$ multibeam sonar operating in an area [9] where moored underwater acoustic transponders provided numerous interference signals likely to challenge any bottom detection algorithm.

In the following, we describe the processing steps applied to each phasor in a ping, and in a continuous sequence of pings. Then, we compare the results of our adaptive scheme to those obtained with a fixed bandwidth filter. We limit our discussion to the estimation of "raw" arrival time-angle pairs for bottom echoes, and our results remain qualitative, pending quantification of the estimation errors.

\section{PHASOR PROCESSING}

The SeaBat 8111ER multibeam echo-sounder operates at $100 \mathrm{kHz}$ with two cylindrical arrays, whose axes are parallel and aligned fore-aft. One array transmits a $1.5^{\circ} \times$ $150^{\circ}$ (fore-aft $\times$ athwartships) fan beam, and the staves of the other array are used to form 101 beams, each $15^{\circ} \times 1.5^{\circ}$ (fore-aft $\times$ athwartships) wide, and spaced $1.5^{\circ}$ apart in the roll plane. The product of the transmit and receive beams produces beams with constant beamwidth, whose directions are fixed in the arrays' reference frame. Adding instantaneous roll angles to these angles places the beams in a vertical reference frame. For this work, the 101 beams are actually phasors output by a split aperture processor, in which each beam formed by one sub-aperture is multiplied by the complex conjugate of the beam formed in the same direction by the other sub-aperture. The base-banded magnitude and phase time series of the 101 phasors were sampled at $5 \mathrm{kHz}$ and recorded with a Reson 6042 processor

Each phasor's magnitude $(M)$ and phase $(\phi)$ time series were converted to the corresponding sequences of the phasor's in-phase $(I)$ and quadrature $(Q)$ components:

$(I, Q)=(M \cos (\phi), M \sin (\phi))$,

which were then filtered independently with identical low-pass filters. Note that this conversion step could have been avoided, had the quadrature samples been recorded directly. However, this option was not available in the Reson 6042 processor that we used.

The cutoff frequency of each phasor's low-pass filter was determined by estimating the duration of the received 


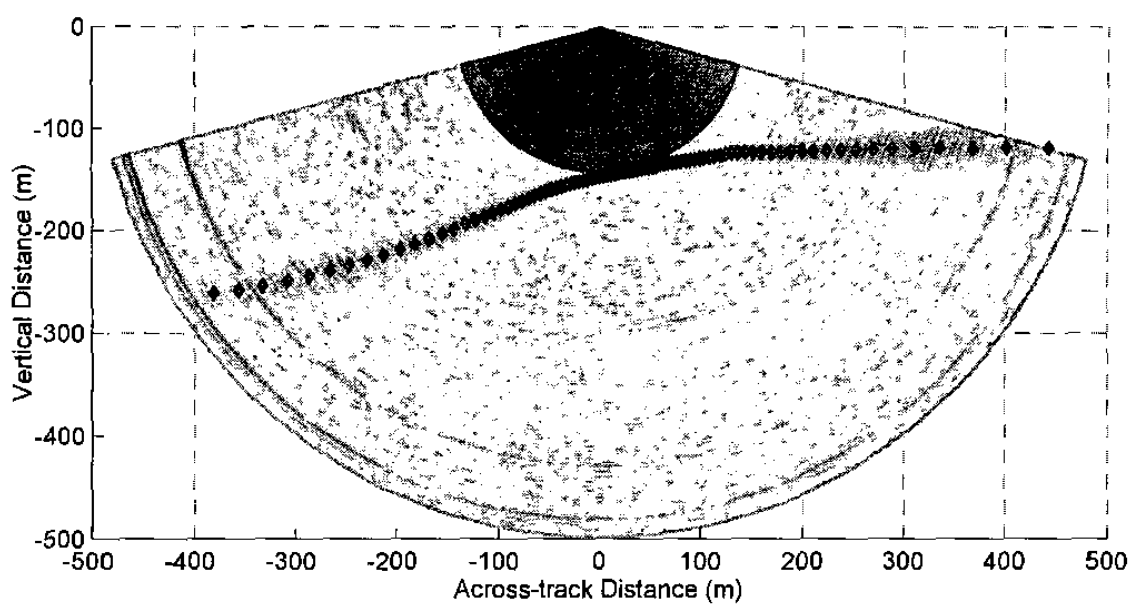

Fig. 1. Bottom detection results for one ping after processing with procedure outlined in Section II. The range determined for each beam $(\downarrow)$ was obtained from either a phase or an amplitude detection.

echo within the $-3 \mathrm{~dB}$ beam width. Phasors with a calculated cutoff frequency exceeding half the $5 \mathrm{kHz}$ sampling frequency were not filtered. Although they are less computationally efficient than infinite impulse response (IIR) filters, we chose finite impulse response (FIR) low-pass filters because of their phase linearity and inherent stability. FIR filter coefficients were calculated using a 41-sample Chebyshev window with $40 \mathrm{~dB}$ of relative sidelobe attenuation ( $-6 \mathrm{~dB}$ attenuation at the cutoff frequency).

The filter's group delay was removed by filtering each sequence twice: once along ascending indices, and once along descending indices. Thus, the amplitude of the

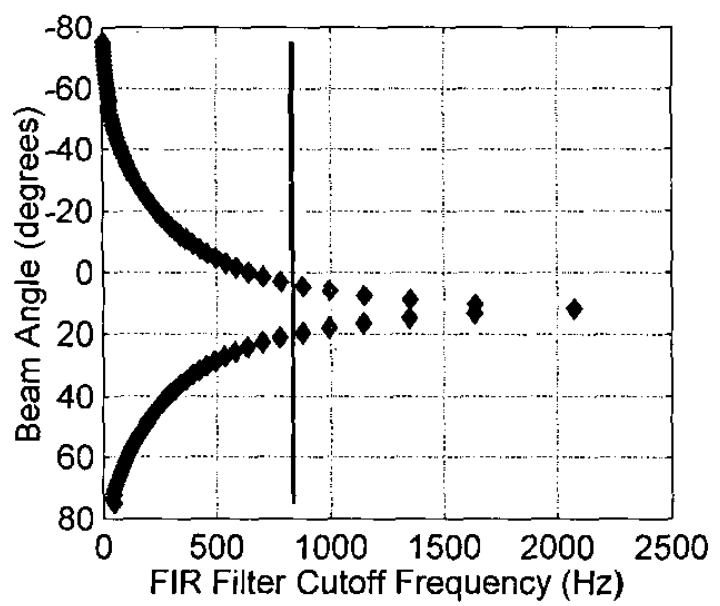

Fig. 2. FIR Chebyshev filter cutoff frequency for each beam (phasor) angle. The solid vertical line at $833.3 \mathrm{~Hz}$ marks the cutoff frequency of a filter whose number of coefficients equals the number of samples in 5 transmitted pulse lengths ( $\tau=241 \mu \mathrm{s}$ ). The first bottom echo from the previous ping was received at $12^{\circ}$, which is the beam angle with the highest filter cutoff frequency $(2074.7 \mathrm{~Hz})$ in this figure. filtered sequence was modified by the square of the filter's magnitude response. Following the filtering operation, each phasor's quadrature samples were converted back to magnitude and phase sequences.

The next step includes two magnitude thresholding operations. First, in each phasor's magnitude sequence, identify the samples exceeding the standard deviation of all samples in the sequence. Second, in each magnitude time slice across all 101 phasors, identify the samples exceeding the standard deviation of the 101 magnitude samples. Those samples identified in both thresholding operations delimit the range window in which to search for the start and end of the bottom backscatter time series for each phasor, and the bottom detection procedure consists in estimating a time and angle of arrival from such bounded time series. Alternatively, the range window could be determined using information on the bottom topography from the previous ping.

The bottom detection procedure is completed in three steps: a concurrent phase and amplitude detection, an amplitude only detection, and setting filter parameters for the next ping. For each phasor, the sign of the phase sequence is adjusted to ensure an overall positive phase slope. Then, searching from the leading edge of the range window identified by the magnitude thresholding operations, the index of the first sample with a phase value within $\pm 5 \%$ of $-\pi / 2$ is marked. The search continues until a sample with a phase value within $\pm 5 \%$ of $\pi / 2$ is found inside the range window, or the trailing edge of the range window has been reached.

The phase slope of the selected samples is checked to ensure that a phase discontinuity has not been selected at either end. If the slope exceeds a preset threshold, the search procedure is repeated until all samples within the range window have been checked.

If a quasi-linear phase sequence containing at least $N$ samples is found within the $\pm \pi / 2$ limits, a robust linear regression (e.g., MatLab ${ }^{\otimes}$ function 'robustfit') is applied to determine the zero crossing, which yields the time of arrival of the bottom echo received at that phasor's beam angle. 
In addition, a conventional magnitude-weighted mean-time detection [10] is computed with the corresponding $N$ samples of the phasor's filtered magnitude sequence.

Two iterations of weighted mean-time detection are performed on phasors' sequences without a valid phase detection. The first iteration uses the time-angle pair of the first bottom echo detected in the previous ping. A pairwise search for adjacent phasors with and without a valid phase detection is performed, starting with the outermost phasor in the ping. Assuming a locally flat seafloor across the corresponding selected beam pair, the range to the center of the current phasor is estimated from the adjacent phasor with a valid phase detection, and the magnitude samples within the $-3 \mathrm{~dB}$ beam width are used to compute an initial estimate of the magnitude-weighted mean-time of arrival.

When all phasors have a valid phase or amplitude detection, the amplitude detection procedure is redone using the time-angle pair of the first bottom echo detected in the current ping.

The last step in the bottom detection procedure is to use
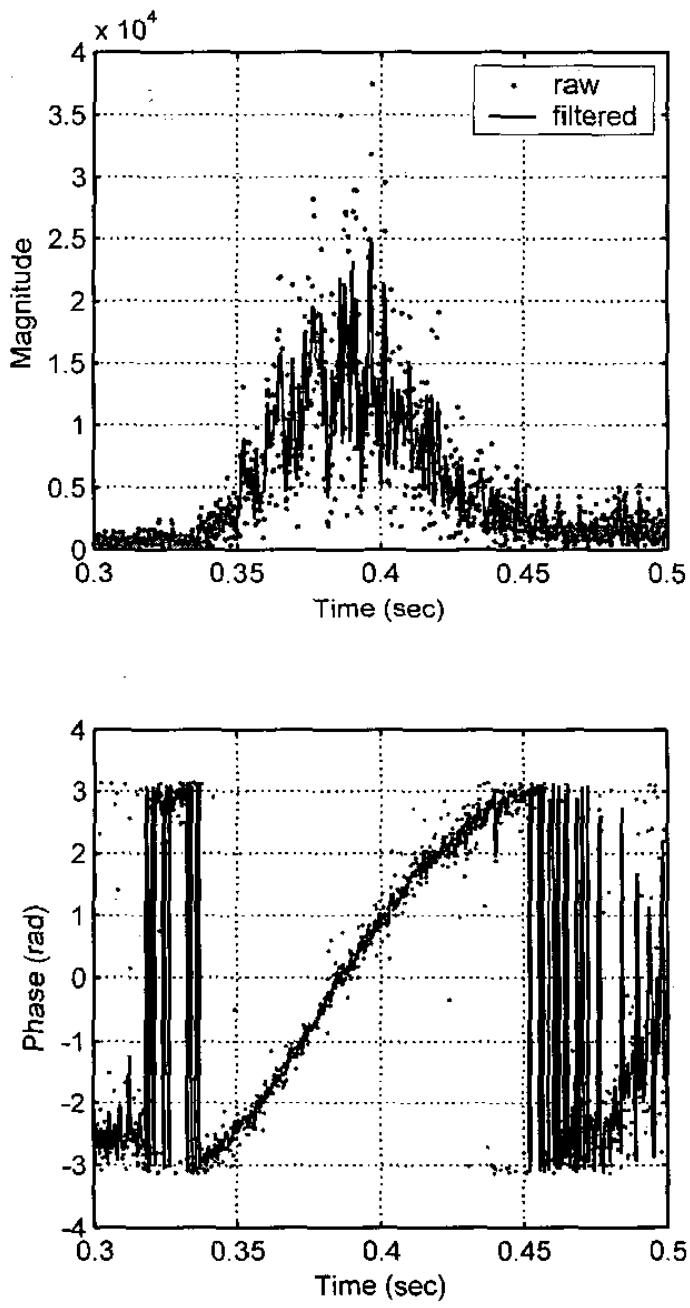

(a) all accepted bottom detections across the swath of the current ping to set the cutoff frequency of each filter for the upcoming ping. For each beam, the duration $t_{b}$ of the bottom echo is estimated from the detected slant range $R$ and angle of arrival $\theta$, the $-3 \mathrm{~dB}$ half beamwidth $\beta$, and the transmitted pulse length $\tau$, assuming a locally flat and horizontal bottom:

$t_{b}=R \cos (\theta)\left[\cos (\theta+\beta)^{-1}-\cos (\theta-\beta)^{-1}\right]+2 \tau$

For beams without a bottom detection, e.g. beams corresponding to $-75^{\circ}$ to $-55^{\circ}$ in Fig. 1 , the duration of the echo is extrapolated using the range and angle of the first received echo in the current ping.

\section{DISCUSSION}

A series of ninety consecutive pings were processed using the procedure outlined in Section II. The results obtained on a representative ping, with a sloping bottom and interferences from external sound sources, are shown
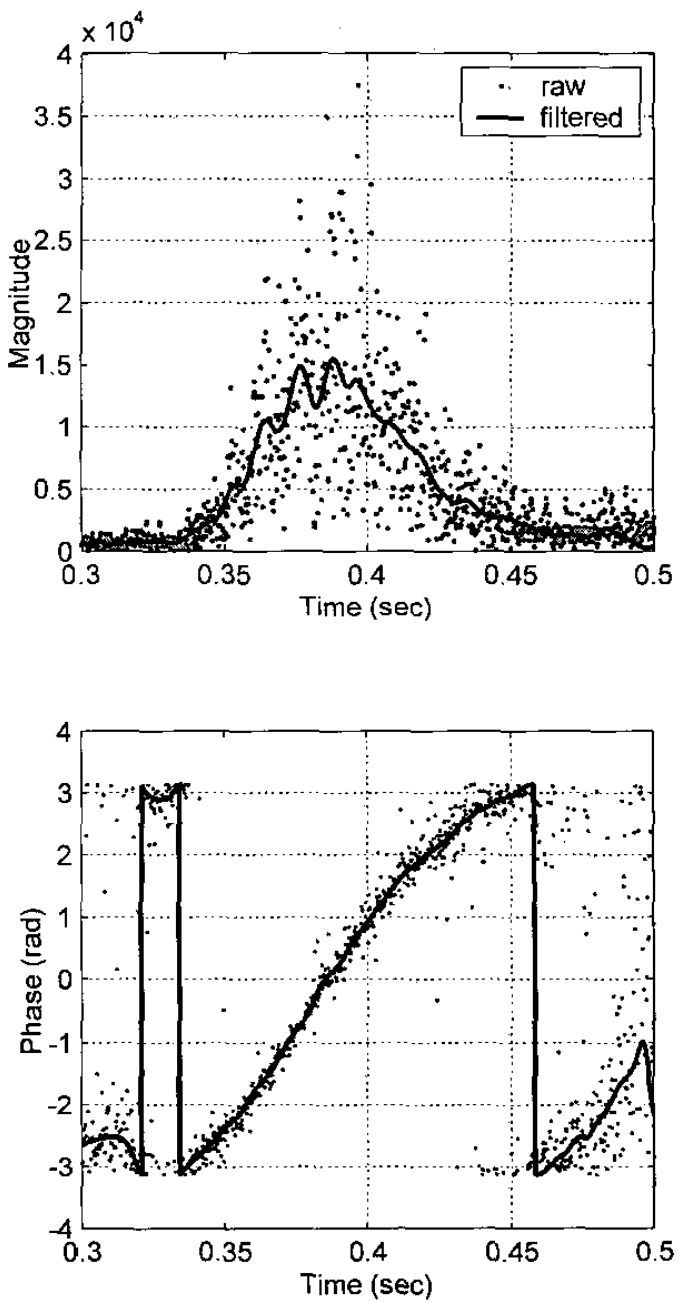

(b)

Fig. 3. Comparison of the (a) fixed and (b) variable filtered magnitude and phase data time series for the beam at an angle of 66 degrees. In this case, the fixed bandwidth filter had a cutoff frequency of $833.3 \mathrm{~Hz}$ and the variable bandwidth filter had a cutoff frequency of $70.1 \mathrm{~Hz}$. 


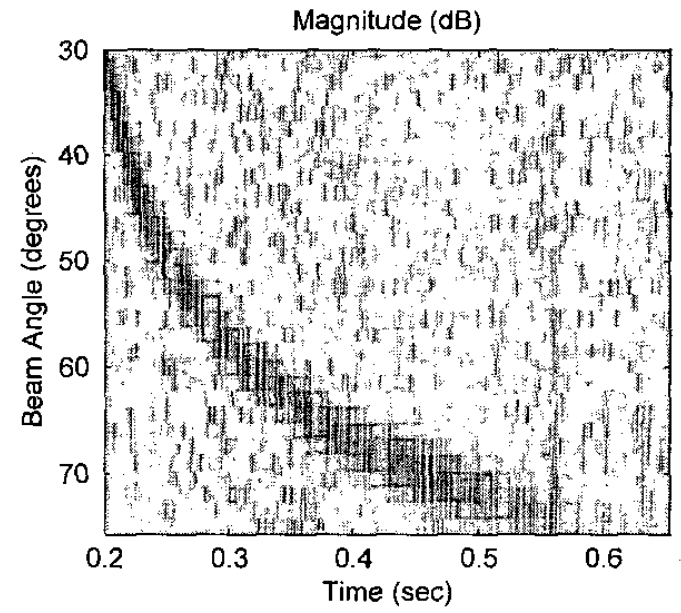

(a)

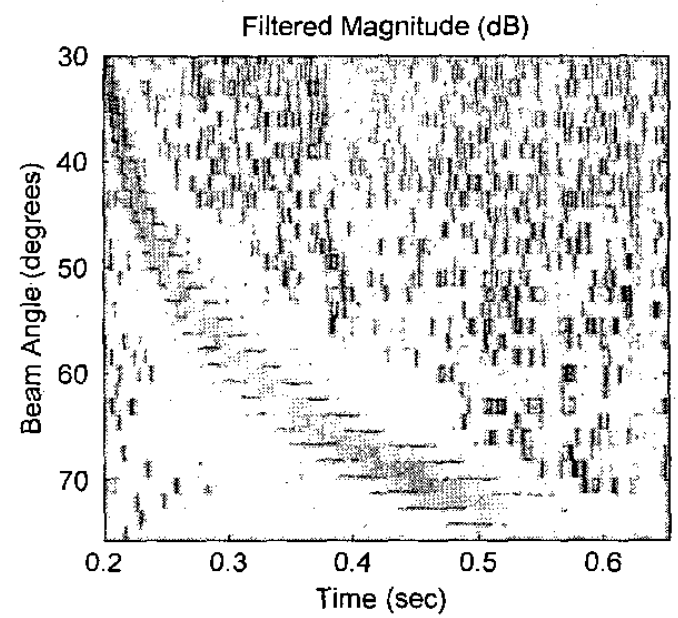

(c)

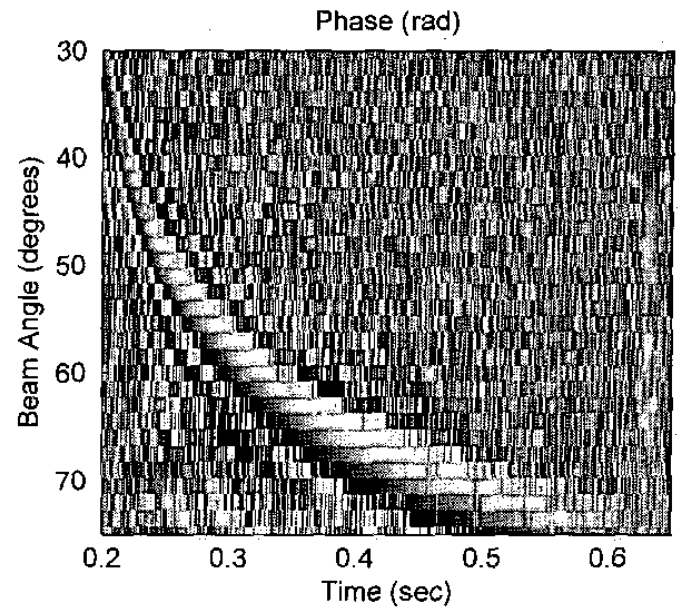

(b)

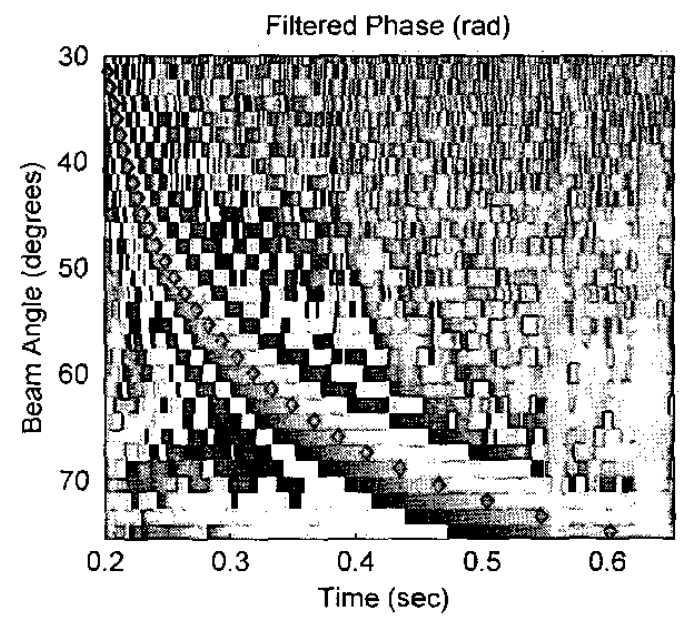

(d)

Fig. 4. Magnitude (a) and phase (b) of a representative ping prior to filtering with a low-pass FIR Chebyshev filter with cutoff frequencies as shown in Fig. 1. Discrete noise spikes are observed in both plots at $0.56 \mathrm{~s}$ and $0.63 \mathrm{~s}$. The filtered magnitude is shown in (c). Shown in (d) is the filtered phase with the range determined for each beam $(\bullet)$ obtained from phase detection superimposed.

in Fig. 1. Bottom detections for all beams in the ping are shown as black diamond symbols superimposed on the filtered magnitude time series data. The magnitude data and bottom detections have been converted from range and angle, to across-track distance and vertical distance (depth in meters), assuming a constant sound speed of $1530 \mathrm{~m} / \mathrm{s}$ and no refraction. For this test case, the algorithm was not affected by the noise spikes or the multipath echoes. This limited data set also illustrates that the algorithm performs well on a sloping seafloor. The water depth across the swath ranges from approximately $120 \mathrm{~m}$ to $260 \mathrm{~m}$.

The low-pass FIR Chebyshev filter cutoff frequencies used for each beam in this ping are shown in Fig 2 . For comparison purposes, the cutoff frequency of a constant bandwidth filter designed based on the number of samples in five pulse lengths, is also shown in the figure.

The oblique outer beams, which are generally the beams with the lowest signal to noise ratio, derive the most benefit from a variable bandwidth filtering approach. Beams within approximately $\pm 10^{\circ}$ of the first received echo have a cutoff frequency close to the fixed bandwidth cutoff frequency $(833.3 \mathrm{~Hz})$ shown in Fig. 2. Filters applied to the outer beams have cutoff frequencies less than one-fiftieth of this fixed bandwidth cutoff frequency.

The reduction in noise realized with the variable bandwidth filter approach is illustrated in Fig. 3, where the magnitude and phase sequences of a beam pointing at $66^{\circ}$ are filtered with (a) the fixed $833.3 \mathrm{~Hz}$ bandwidth low-pass FIR Chebyshev filter, and (b) with a FIR Chebyshev filter whose cutoff frequency was set at $70.1 \mathrm{~Hz}$. The improved noise reduction with the lower cutoff frequency is readily apparent.

The improvement across the entire swath is shown in Fig. 4. For display clarity, beam angles from $-75^{\circ}$ to $30^{\circ}$ have been omitted. In Fig. 4(a), the unfiltered magnitude of a representative ping is shown. Two unwanted noise 
spikes can be seen at arrival times of 0.56 and $0.63 \mathrm{~s}$. The noise spikes are also apparent in the corresponding unfiltered phase data shown in Fig. $4(\mathrm{~b})$. The filtered magnitude and phase time series data are shown in Figs. 4(c) and Fig. 4(d), respectively, after filtering with a low-pass FIR Chebyshev filter with a different cutoff frequency for each beam as shown in Fig. 2 .

\section{CONCLUSIONS}

A variable bandwidth filtering approach has been shown to reduce the noise in multibeam sonar data that hampers the bottom detection process. Information from the previous ping is used to calculate the filter coefficients for the next ping with the bandwidth of the filter varying across the swath width. The filter cutoff frequency was determined by estimating the duration of the received echo within the $-3 \mathrm{~dB}$ beam width. Beams with short time echoes have a broad filter bandwidth; beams with long time echoes have a narrow filter bandwidth.

The approach was evaluated with Reson SeaBat 8111ER multibeam echo sounder data. Although the results of only a representative ping have been presented here, excellent results were obtained for the entire sequence of 90 pings, thus justifying the investigation of a real-time implementation. However, with ping rates as high as $40 \mathrm{~Hz}$, the procedure must be heavily optimized for processing speed. One obvious option is to take advantage of the computational efficiency of IIR filters over FIR filters, achieved through much lower filter orders for comparable bandwidths. The disadvantage is that IIR filters may experience stability issues and have nonlinear phase characteristics. The nonlinear phase distortion can be overcome by filtering in both directions as was done with the FIR filters used in this study.

\section{Acknowledgments}

The authors are grateful to Burr Bridge of Reson, Inc. for acquiring the phasor data while at sea.

\section{REFERENCES}

[1] C. de Moustier, "Signal processing for swath bathymetry and concurrent seafloor acoustic imaging," in Acoustic Signal Processing for Ocean Exploration, $\mathrm{J}$. M. F. Moura and I. M. G. Lourtie, Eds. NATO ASI Series, Kluwer, pp. 329-354, 1993.

[2] E. Hammerstad and F. Pohner, "Ultra wide swath deep sea interferometric multibeam echo sounder," Proc. IEEE Oceans '91, vol. 2, pp. 743-749, 1991.

[3] E. Hammerstad, S. Asheim, K. Nilsen, and H. Bodholt, "Advances in multibeam echo sounder technology," Proc. IEEE Oceans '93, pp. 1482-1487, 1993.

[4] L. Yang and T. Taxt, "Multibeam sonar bottom detection using multiple subarrays," Proc. IEEE Oceans '97, vol. 2, pp. 932-938, 1997.

[5] T. C. Gallaudet, "Shallow water acoustic backscatter and reverberation measurements using a $68-\mathrm{kHz}$ cylindrical array," Ph. D. Dissertation, MPL, Scripps Institution of Oceanography, Univ. of California, San Diego, 2001.

[6] F. LeClerc, "Performance of angle estimation methods applied to multibeam swath bathymetry," Proc. IEEE OCEANS '94, vol. 3, pp. 231-236, 1994.
[7] X. Lurton, "Acoustical measurement accuracy modeling for bathymetry sonar systems," Proc. Canadian Hydrographic Conf., Toronto, CA, May 28-31, 2002.

[8] M. A. Masnadi-Shirazi, C. de Moustier, P. Cervenka, and S. H. Zisk "Differential phase estimation with the SeaMARC II bathymetric sidescan sonar system," IEEE J. Oceanic Eng., vol. 17, no. 3, pp. 239-251, July 1992.

[9] M. P. Porter and The Kauai HFX Group, "The Kauai experiment," in Int. Conf. on High-Frequency Ocean Acoustics, La Jolla, CA, Mar. 2004

[10] H. K. Farr, "Multibeam bathymetric sonar: Sea Beam and Hydrochart," Marine Geodesy, vol. 4, no. 2, pp. $77-93,1980$ 